\title{
Stability of Nuclei
}

\author{
Arbind Kumar Mallik \\ Department of physics,Tribhuwan Multiple Campus, Palpa \\ ak_mallik@hotmail.com
}

\begin{abstract}
Nucleus is composed of uncharged neutrons and charged protons, chiefly given the fact that the electrostatic repulsion of the protons tends to disrupt the nucleus? What is the nature of forces that ensure stability between charged and uncharged particles in the nucleus? Attempts have been made to solve this problem both on the experimental and theoretical sides. In this article, a brief statement of these investigations is described. Search for the causes of stability of nuclei have been made in the following four directions, Relative abundance of elements as well as of isotopes that occur in nature, The binding energy per nucleon curve, Decay constant of natural radioactive substances, Magic numbers.
\end{abstract}

\section{Introduction}

The basic assumption made in this study is that elements and isotopes which occur in nature quite abundantly are very stable. There are now two branches of research, (a) the relative abundance of elements with respect to one another and (b) the relative abundance of the different isotopes within each element.

To solve the first problem, an enormous number of chemical analysis of igneous rocks and other geological materials (like earth's crest, a few meteorites, some nebula \& the outermost layers of stars) has been made. The more powerful and convenient method of X-ray analysis has been applied by Hevesy, Noddack and others. As a result of the vast data certain conclusions have been arrived, they are :-

(i) Heavier elements are much rare than lighter ones which means that the light elements are much more stable than heavy ones.

(ii) There is a great predominance of elements of even atomic number $(Z)$ over elements of odd, first pointed out by Harkin, hence called Harkin's rule which has been confirmed by X-ray analysis, even in the case of rare earths. This finding means that atoms of odd $\mathrm{Z}$ are much less stable than those of even $\mathrm{Z}$.
It is found that the relative quantities of the different isotopes are the same for every sample of a given element, from whatever part of the earth's crest or even out of the meteorites it might have been taken. The results obtained may be summarised as follows:-

a. Elements of even atomic number Z, usually posses a much larger number of stable isotopes than the elements of odd $\mathrm{Z}$ which almost never have more than two stable isotopes.

b. In light nuclei the mass number $\mathrm{A}$ is approximately double the atomic number $\mathrm{Z}$, while in heavy nuclei the mass number $\mathrm{A}$ is somewhat higher. This might mean that stability conditions require that the ratio of neutrons to protons must approximately to unity in light nuclei, but become greater than unity in heavy nuclei.

c. Out of the 280 isotopes that occur in nature, 154 are of even $A$ and even $Z$, While there are only four with even A and odd Z. $\left({ }_{1} \mathrm{H}^{2},{ }_{3} \mathrm{Li}^{6},{ }_{5} \mathrm{~B}^{10}\right.$ and $\left.{ }_{7} \mathrm{~N}^{14}\right)$. This means that nuclei with even numbers of protons and neutrons are more stable than those with odd numbers. There are 107 stable isotopes nuclei having odd $\mathrm{A}$, of which 55 have even $\mathrm{Z}$ and 52 having odd $\mathrm{Z}$. Hence it follows that isotopes of even $Z(154+55)$ are much more abundant and hence more stable than those of odd Z $(152+4)$. 


\section{The binding energy per nucleon curve:-}

The binding energy involved in the formation of the nucleus from its constituents is depending on the mass defect. Hence the greater the mass defect, the greater the binding energy, but greater the interchange of energy in the building up of the nucleus, the greater will be the union of the constituent parts in a general sense, hence greater the stability of the nucleus formed, minimum potential energy representing the most stable state. On the other hand, to a small mass defect would correspond a small binding energy and in consequence, the nucleus involved will not be very stable.

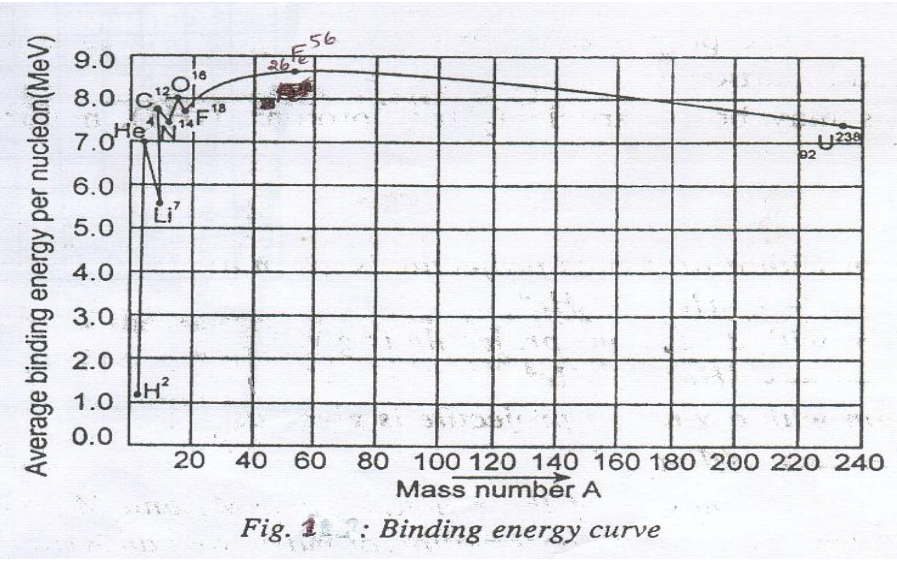

The average binding energy per nucleon is obtained by dividing total binding energy by mass number A. Binding energy per nucleon is plotted as a function of mass number A in fig-1.

Conclusions of nuclear binding energy per nucleon curve are given as:-

a. Nuclei with $\mathrm{A}<30$ have a periodic recurrence of peaks in the binding energy per nucleon for mass numbers which are multiples of four. Each of these nuclei has $\mathrm{Z}=\mathrm{N}$

b. Nuclei of intermediate mass have binding energy per nucleon remain nearly constant i.e, between 8 to 8.79 $\mathrm{MeV}$ per nucleon. These nuclei are the most stable since the greatest amount of energy must be supplied to liberate each of their nucleons.

c. The curve drops slowly to about 7.6 $\mathrm{MeV}$ at the higher mass number $\mathrm{A}=238,\left(\mathrm{U}^{238}\right)$. These nuclei are energetically unstable.

d. In light nuclei $\mathrm{A}<20$, the average binding energy per nucleon is generally low. This is due to the fact that a large fraction of nucleons does not have a saturated structure of exchange forces.

\section{Decay constant of heavy radioactive nuclei:-}

Radioactive atoms are spontaneously unstable. If decay constant $\lambda$ is small the stability is great, since the probability to disintegrate in unit time is small. The dependence of $\lambda$ on the mass number $\mathrm{A}$ as well as on the decay energies has been studied.

The relation between $\lambda$ and decay energy has been investigated by Geiger and Nuttal in the case of $\alpha$ - disintegration and by Sargent for $\beta$-disintegration. In both the cases, a direct proportionality between $\lambda$ and the decay energy has been found although in $\beta$ disintegration the variation is much slower than in $\alpha$ - disintegration. From these experimental data, a general inference can be made, i.e, the great the energy of disintegration the less the stability of the parent nucleus against disintegration.

\section{Magic numbers:}

It is observed that nuclei having either the number of protons $\mathrm{Z}$ or number of neutrons $\mathrm{N}=\mathrm{A}-\mathrm{Z}$ equal to one of the number 2,8,20,50,82 and 126 have a very high stability as compared to their neighbours. These numbers are called magic numbers. Similarly nuclei with $14,28, \& 40$ nucleons are slightly less stable but more stable than the rest. These numbers are called semi magic numbers.

Some experimental evidence for nuclear magic numbers is given as:-

i. It has been experimentally observed that ${ }_{2} \mathrm{He}^{4}$ with $\mathrm{Z}=\mathrm{N}=2$ and ${ }_{8} \mathrm{0}^{16}$ with $\mathrm{Z}=\mathrm{N}=8$ are highly stable. A graph between binding energy per nucleon for ${ }_{20} \mathrm{Ca}^{40}(\mathrm{Z}=\mathrm{N}=20)$ ${ }_{38} \mathrm{Sr}^{88}(\mathrm{Z}=38, \mathrm{~N}=50),{ }_{50} \mathrm{Sn}^{120}(\mathrm{Z}=50, \mathrm{~N}=70),{ }_{58} \mathrm{Ce}^{140}(\mathrm{Z}=58$, $\mathrm{N}=82),{ }_{82} \mathrm{~Pb}^{208}(\mathrm{Z}=82, \mathrm{~N}=126)$

ii. The doubly magic nuclei i.e, which have $\mathrm{Z}$ and $\mathrm{N}$ both magic numbers are particularly tightly bound. For example ${ }_{2} \mathrm{He}^{4},{ }_{8} \mathrm{O}^{16},{ }_{20} \mathrm{Ca}^{40},{ }_{22} \mathrm{~Pb}^{208}$ are more stable nuclei

iii. The binding energy of the next neutron or proton after a magic is very small.

iv. The nuclides whose proton number $\mathrm{Z}$ corresponds to a magic numbers have the highest number of isotopes. For example ${ }_{20} \mathrm{Ca}^{40}(\mathrm{Z}=20)$ has six isotopes whereas the average number of isotopes in that region is about two. Similarly ${ }_{50} \mathrm{Sn}^{120}(\mathrm{Z}=50)$ has 10 isotopes whereas the average number in this region is about 4.

v. The stable end product of the natural radioactive series is 
lead $\left({ }_{82} \mathrm{~Pb}^{208}\right)$. It has $\mathrm{Z}=82$ and $\mathrm{N}=126$ both are magic number.

vi.

The electric quadruple moment of nuclei with protons and neurons equal to magic number is zero. These nuclei are therefore, spherically symmetric because electric quadruple moment measures the departure of nuclear charge distribution from spherical symmetry. Nuclei in the neighbourhood of magic number nuclei have certain value of quadruple moment i.e, these have distorted shape.

The significance of magic number lies in the fact that the corresponding number of protons or neutrons gives a high stability to the nucleus. The presences of magic numbers lead to the development of the shell model of the nucleus by analogy with the closed shells and sub shells in the case of atoms. There is a similarity of stability between magic numbers nuclei and the inert gas atoms. The first, second, third etc shells closures in nuclei occurs at number of protons or neutrons in the nucleus equal to $2,8,20,50,82$, and 126 which are magic numbers. According to Maria Meyer the energy states occupied by the nucleons are as follows:-

\begin{tabular}{|l|l|l|l|}
\hline $\begin{array}{l}\text { Nuclear } \\
\text { Shell }\end{array}$ & spectroscopic terms & $\begin{array}{l}\text { Nucleons in } \\
\text { Shell }\end{array}$ & $\begin{array}{l}\text { Total } \\
\text { number } \\
\text { of nucleons }\end{array}$ \\
\hline I & $\left(1 \mathrm{~s}_{1 / 2}\right)^{2}$ & 2 & \\
II & $\left(2 \mathrm{p}_{32}\right)^{4}\left(2 \mathrm{p}_{1 / 2}\right)^{2}$ & 6 & 2 \\
IIa & $\left(3 \mathrm{~d}_{5 / 2}\right)^{6}$ & 6 & 8 \\
III & $\left(2 \mathrm{~s}_{1 / 2}\right)^{(2}\left(3 \mathrm{~d}_{3 / 2}\right)^{4}$ & 6 & 14 \\
IIIa & $\left(4 \mathrm{f}_{7 / 2}\right)^{8}$ & 8 & 28 \\
IV & $\left(3 \mathrm{p}_{32}\right)^{4}\left(4 \mathrm{f}_{5 / 2}\right)^{6}\left(3 \mathrm{p}_{1 / 2}\right)^{2}\left(5 \mathrm{~g}_{92}\right)^{10}$ & 8 & 50 \\
V & $\left(5 \mathrm{~g}_{7 / 2}\right)^{8}\left(4 \mathrm{~d}_{5 / 2}\right)^{6}\left(4 \mathrm{~d}_{32}\right)^{4}\left(3 \mathrm{~s}_{1 / 2}\right)^{2}(6 \mathrm{~h}$ & 22 & 82 \\
VI & $11 / 2)^{12}$ & 32 & 126 \\
$\left(6 \mathrm{~h}^{1 / 2}\right)^{10}\left(5 \mathrm{f}_{7 / 2}\right)^{8}\left(5 \mathrm{f}_{5 / 2}\right)^{6}\left(4 \mathrm{p}_{3 / 2}\right)^{4}$ & 44 & \\
$\left(4 \mathrm{p}_{1 / 2}\right)^{2}\left(7 \mathrm{i}_{132}\right)^{14}$ & 44 & \\
\hline
\end{tabular}

Here II a and IIIa sub-shell are closed with 14 and 28 nucleons which are semi magic numbers.

\section{Theoretical study of stability of nucleus}

\section{Heisenberg: Majorana theory of "exchange" forces}

Heisenberg and Majorana proposed in 1933 a theory of nuclear forces based on the proton neutrons constitution of the nucleus and a suitable combination of the liquid drop and shell models, which was able to explain several of the experimentally observed facts concerning the stability of nuclei satisfactorily though not completely.

Majorana also explain the electrostatic repulsive forces between the protons in the nucleus. It can be shown that the positive potential energy due to this repulsive force is $0.25 \mathrm{MeV}$ which is indeed, negligible compared with the average binding energy per nucleon $(8.5 \mathrm{MeV})$. However, because of the saturation character of the intranuclear forces. the Coulomb repulsion between the protons becomes important for heavy nuclei. The Coulomb forces show no saturation. Hence the total energy of the Coulomb interaction need not be proportional to the mass number A. In fact, it can be shown that the electrostatic energy is proportional $A^{5 / 3}$. Hence the relative importance of the electrostatic force increases, with increasing mass number as $\mathrm{A}^{2 / 3}$. The binding energy per nucleon will therefore decrease, on account of the electrostatic force; with increasing mass number as is actually the case with heavier nuclei. This work in the opposite direction from the surface tension effect.

Yukawa in 1935, in an attempt to remove the difficulties of Heisenberg- Majorana theory of "exchange forces" modified the theory known as Yukawa "meson field theory". According to the meson theory of nuclear forces, all nucleons consist of identical cores surrounded by a 'cloud' of one or more mesons. Meson may be neutral or may have a positive or negative charge. It is believed that due to the different types of composition of cloud, the neutrons and protons are different. Yukawa assumed that л-meson is exchanged between the nucleons and this is responsible for the nuclear binding energy. The forces that act between two neutrons and two protons are the result of exchange of neutral mesons $\left(л^{0}\right)$ between them. The nuclear force between a neutron and a proton is due to the exchange of charged mesons ( $\pi^{+}$and $\left.\pi^{-}\right)$between them. Thus a neutron emits a ${ }^{-}$meson and is converted into a proton i.e, $\mathrm{n} \quad \mathrm{P}+\pi^{-}$ 
The absorption of $\pi^{-}$meson by the proton converts into a neutron.

i.e, $\quad \mathrm{P}+\pi^{-} \mathrm{n}$ or proton emits $\pi^{+}$meson and converts into a neutron and a neutron receiving this $\mathrm{J}^{+}$meson becomes a proton.

$\begin{array}{lll}\mathrm{Pn}+\pi^{+} & \mathrm{n}+\pi^{+} & \mathrm{P}\end{array}$

Thus in the nucleus of an atom, attractive forces exist between

i. $\quad$ Proton and proton

ii. Proton and neutron

iii. Neutron and neutron.

$\left(\pi^{\circ}, \pi^{+}\right.$and $\left.\pi^{-}\right)$.

For stable nuclides having atomic number $Z<20, N=Z$, for $Z>20$ the number of neutrons has to be greater than $Z$ for stability of the nucleus. This is because for stable nuclei having large values of $Z$. The Coulomb force of repulsion between the protons becomes very large. In order to compensate the large repulsion force there has to be greater number of neutrons.

\section{Reference:}

These forces of attraction are much larger than the electrostatic force of repulsion between the protons, thus giving stability to the nucleus.

1. Murugeshan R. and Sivaprasth K; 2007. Modern physics, S.Chand \& Co. Ltd. New Delhi.

2. Kaplan I; 1962. Nuclear physics $2^{\text {nd }}$ ed. Oxford \& IBH publishing Co. Pvt. Ltd.

\section{Conclusion}

It is fount that nuclear forces holding the nucleons together inside the nucleus, which are short range, basically very strong about $10^{38}$ times as strong gravitational forces, charge independent (i.e, $n-p=$ $\mathrm{n}-\mathrm{n}=\mathrm{P}-\mathrm{P})$, charge symmetric, spin dependent and non- central forces.

These forces exist between nucleons due to exchange of л- mesons

3. Arora C.L; 1999. Nuclear physic, S.Chand \& Co. Ltd. New Delhi.

4. Rajam J.B; 1999. Atomic physics, S. Chand \& Co. Ltd. New Delhi.

5. Tayal D.C; 2002. Nuclear physics, Himalaya publishin house, Mumbai.

6. Duggal B.D and Chhabra C.L; 1990. Fundamentals of Modern Physics, Shoban Lal Nagin Chand \& Co. Delhi. 